\title{
Review
}

\section{High throughput screening technologies for ion channels}

\author{
Hai-bo YU*, Min LI, Wei-ping WANG, Xiao-liang WANG*
}

State Key Laboratory of Bioactive Substances and Functions of Natural Medicines, Institute of Materia Medica, Chinese Academy of Medical Sciences and Peking Union Medical College, Beijing 100050, China

\begin{abstract}
Ion channels are involved in a variety of fundamental physiological processes, and their malfunction causes numerous human diseases. Therefore, ion channels represent a class of attractive drug targets and a class of important off-targets for in vitro pharmacological profiling. In the past decades, the rapid progress in developing functional assays and instrumentation has enabled high throughput screening (HTS) campaigns on an expanding list of channel types. Chronologically, HTS methods for ion channels include the ligand binding assay, flux-based assay, fluorescence-based assay, and automated electrophysiological assay. In this review we summarize the current HTS technologies for different ion channel classes and their applications.
\end{abstract}

Keywords: ion channels; high throughput screening; ligand binding assay; flux-based assay; fluorescence-based assay; automated electrophysiological assay; drug discovery

Acta Pharmacologica Sinica (2016) 37: 34-43; doi: 10.1038/aps.2015.108; published online 14 Dec 2015

\section{Introduction}

Ion channels are a very important membrane protein family involved in a variety of fundamental physiological processes. Their malfunction causes a variety of human diseases. Therefore, ion channels represent a class of attractive drug targets ${ }^{[1-4]}$ and a class of important off-targets for in vitro pharmacological profiling ${ }^{[5]}$. Traditionally, patch clamp electrophysiology is the gold standard for ion channel studies. However, the method is labor-intensive with a low throughput and requires highly trained staff to perform the experiments. Ion channels are difficult targets to be investigated using high throughput approaches, which hinders the use of ion channels compared with other targets. Recently, the rapid progress in developing functional assays and instrumentation has enabled high throughput screening (HTS) campaigns on an expanding list of channel types. Consequently, HTS was designed to identify active compounds for ion channel targets, which are of great interest to academic and industrial researchers. The methodologies for studying ion channels can be divided into non-electrophysiological and electrophysiological methods. This review will summarize the current technologies and commonly used screening methods for different ion channel classes.

\footnotetext{
* To whom correspondence should be addressed.

E-mail haiboyu@imm.ac.cn (Hai-bo YU); wangxl@imm.ac.cn (Xiao-liang WANG)

Received 2015-09-15 Accepted 2015-10-12
}

\section{High throughput screening technologies}

In the past, HTS methods for ion channels have been extensively developed and applied to most ion channels. In chronological order, the approaches include: the ligand binding assay, flux-based assay, fluorescence-based assay and automated electrophysiological assay.

\section{Ligand binding assays}

Ligand binding assays have been widely used to screen for ion channel modulators. However, these assays are not considered as functional assays because they detect the binding affinity of a compound to an ion channel rather than the ability of altering channel function. Ligand binding assays require a previous knowledge of the target binding sites and of the formation of a radio-labeled ligand which is specific to those binding sites. Activity of the test compound is indicated by the displacement of the labeled ligand. Consequently, conventional instrumentation may be used, in which throughput represents its major strength. Because the method only discovers compounds that influence radioligand binding, it misses allosteric modulators of ion channels ${ }^{[6-9]}$. Binding assays identify affinity data but do not identify the functional change of ion channels. For example, an agonist cannot be distinguished from an antagonist in a binding assay. Secondary assays are necessary to determine if the compound is an agonist, antagonist or neither. Furthermore, the scope of binding assay is limited by the availability and affinity of radio-labeled ligands ${ }^{[7-9]}$. 
The sensitivity of a binding assay is often determined by the affinity of a known labeled ligand. A high-affinity ligand may not allow the detection of weak binders. However, the use of a low-affinity ligand can lead to an increased detection of nonspecific binding. When the ligand affinity is within a certain range (eg, from nano- to micro-molar concentration), the $\mathrm{IC}_{50}$ values obtained from binding assays have a reasonable correlation ${ }^{[10]}$ with those obtained from patch clamping. Thus far, the assay format has been rarely used for general screening but is still good for identifying modulators specific to some given ligands.

\section{Flux-based assays}

Ion flux assay has been successfully applied to directly access a functional change of ion channel activity. Radioactive isotopes have been used to trace the cellular influx or efflux of specific ions, such as ${ }^{22} \mathrm{Na}^{+},{ }^{45} \mathrm{Ca}^{2+}$ and ${ }^{86} \mathrm{Rb}^{+}$, for the studies of $\mathrm{Na}^{+}, \mathrm{Ca}^{2+}$ and $\mathrm{K}^{+}$channels, respectively. A commonly used assay format is the ${ }^{86} \mathrm{Rb}^{+}$efflux for $\mathrm{K}^{+}$channels or non-selective cation channels. In this format, the cells that express the ion channel of interest are incubated with a buffer that contains ${ }^{86} \mathrm{Rb}^{+}$for several hours before they are washed and stimulated with an agonist to allow for ${ }^{86} \mathrm{Rb}^{+}$efflux. Then the cells and supernatant are collected for radioactive counting ${ }^{[11,12]}$. However, radioactive-efflux assays suffer from the inconvenience and cost associated with the handling of radioactive materials. Additionally, it is necessary to use different radioisotopes for channels that are selective for different ions. Therefore, a nonradioactive $\mathrm{Rb}^{+}$efflux assay was developed that uses atomic absorption spectroscopy to detect rubidium ${ }^{[13]}$. The flux assay is a format preferred by many screening laboratories because it measures ionic flux that better correlates with the activity ${ }^{[14-16]}$. This assay technology is widely applied in the pharmaceutical industry for both drug discovery and hERG-related drugsafety screening to identify potential QT liabilities that might cause lethal arrhythmias ${ }^{[6,17]}$. However, these assays have the disadvantages of low temporal resolution (typically from seconds to minutes), uncontrolled membrane potential, less information content compared with voltage-clamping and lower throughput compared with fluorescence-based assays. Furthermore, this assay generates a very weak signal for some ion channels, which requires a high level of channel expression to achieve an acceptable signal-to-noise ratio.

\section{Fluorescence-based assays}

Fluorescence-based methods do not directly measure ionic current. Rather, they measure either the membrane-potentialdependent or ion-concentration-dependent changes of fluorescence signals as a result of ionic flux. Because fluorescencebased methods produce a robust and homogeneous cell population measurement, these assays are similar to those for other protein classes. Therefore, more instrument choices and expertise are available. Consequently, these assays are relatively easy to implement and to optimize to achieve a higher throughput.

\section{Voltage-sensitive dye assays}

Fluorescent voltage-sensitive dyes measure voltage changes across the cellular membrane using either the potential-dependent accumulation and redistribution ${ }^{[18]}$ or the fluorescence resonance energy transfer (FRET) mechanism ${ }^{[19]}$. Oxonol derivatives, such as bis-(1,3-dibutylbarbituric acid) trimethine oxonol [DiBAC 4 (3)], are lipophilic and negatively-charged dyes that display an increased quantum yield in an aqueous environment upon binding to hydrophobic intracellular molecules instead of weak fluorescence. Because the change in fluorescence occurs minutes after the change in membrane potential, these dyes are best suited to detect a steady state instead of kinetic changes of membrane potential. These dyes cannot detect second and sub-second resolution changes of membrane potential. In contrast, the FMP dye, another anionic membrane potential dye from the FLIPR Membrane Potential Assay Kit (Molecular Devices, Sunnyvale, CA, USA), provides a faster response time (in tens of seconds) than $\mathrm{DiBAC}_{4}(3)^{[20]}$ and is good for detecting kinetic signal changes.

The lipophilic character of $\operatorname{DiBAC}_{4}(3)$ and the FMP dye is associated with a lack of membrane selectivity, which indicates that these voltage-sensitive dyes can respond to a membrane potential change from both the plasma membrane and the endo-membrane. For FRET-based assays, the negatively charged, membrane-soluble oxonol dyes (bis-(1,3-dialkylthiobarbituric acid) trimethine oxonol [DiSBAC $\left.C_{n}(3)\right]$ ), are used as voltage-sensing FRET acceptors. The FRET donors are coumarin-tagged phospholipids (CC2-DMPE) that are integrated into the outer leaflet of the membrane when loaded into the cells. An increase or decrease of FRET in response to membrane hyperpolarization or depolarization produces fast, ratiometric changes. The ratiometric nature of the assay helps to eliminate many artifacts associated with DiBAC assays. Unlike DiBAC assays, the use of phospholipid-anchored FRET donor restricts the location of FRET in the plasma membrane, which ensures that the measurement of potential changes occur at the cell membrane rather than in other subcellular compartments, such as the mitochondria. In addition, FRET-based voltage sensors produce sub-second temporal resolution, which allows for kinetic reading. Because the mobile oxonol molecules are charged, a dye-concentration-dependent dye-current may interfere with the change of membrane potential caused by the ionic current through the ion channels, especially when the current is less than a few hundred pico-amperes. One way to reduce this interference is to use less dye, which may cause a decrease in the signal-to-noise ratio if the dye concentration is below a certain limit.

\section{Ion-specific fluorescent probes}

The assays that measure intracellular ionic concentrations are widely used in research and pharmaceutical screenings for ion channels. Different ion-specific synthesized probes have been developed, such as calcium indicator dyes (Fura2, Fluo-3, Fluo-4 ${ }^{[21,22]}$, potassium indicator dyes (FluxOR ${ }^{[23,24]}$ and $\mathrm{PBFI}^{[25]}$ and sodium indicator $\mathrm{SBFI}^{[26]}$. In addition to the 
synthesized ion indicator dyes, a genetically-coded fluorescent protein can be used as an indicator of ion channels. Specifically, mutations of YFP (H148Q and I152L) further increase the YFP-halide sensitivity, which is suitable for chlorideinvolved channel assay ${ }^{[27-29]}$. These indicators are usually used in conjunction with a FLIPR-type kinetic fluorescent reader to achieve high throughput, low-noise detection of both absolute levels and changes of cytosolic corresponding ionic concentrations.

Overall, ion channel assays that use ion indicator dyes are largely limited to the availability of high performance ionspecific indicators. To date, calcium indicators are most extensively utilized and provide a robust performance that can be used for HTS of calcium channels or non-selective cation channels ${ }^{[30-33]}$.

\section{Automated electrophysiological assays}

Patch-clamp has been widely considered as the gold standard to directly record ion channel activity. This technology provides high quality and physiologically relevant data of ion channel function at the single cell or single channel (within a small patch of membrane) level. For pharmacological testing of compounds, it provides a standard for measuring the potency of compound-channel interactions. Although the conventional patch-clamp offers a direct, information-rich and real-time method to study the channel function, it has very low throughput and a labor-intensive nature, which requires highly skilled and trained personnels. Over the last decade, the development of an automated planar patch clamp has been a breakthrough. Many automated electrophysiology platforms have been developed and are commercially available. They either provide a giga-ohm seal quality of data comparable to a manual electrophysiology recording, or they rely on the use of a perforated patch clamp technique with higher throughput that does not compromise data quality and pharmacology.

The IonWorks platform was the first commercially available automated electrophysiological screening platform to gain widespread utility and validation in the field. It is now available in its second generation, the IonWorks Quattro ${ }^{\mathrm{TM}}$, and a more recent generation, the IonWorks Barracuda ${ }^{\mathrm{TM}}{ }^{[34-36]}$. The system operates in a 384-well format in two modes, single-hole mode and population patch clamp mode (PPC). In single-hole mode, one cell per well is recorded in a 384-well plate. In PPC mode, the instrument reports average currents from recordings of up to 64 cells per well. The data recorded using singlehole mode achieved a lower success rate with a considerable well-to-well variability. However, it is the preferred mode for clonal screening and selection during cell line development ${ }^{[37]}$. PPC mode provides an improved data consistency and success rate in the measurement of ionic currents ${ }^{[34]}$. A common limitation has been observed for lipophilic compounds with right-shifted potencies due to the use of plastic materials for the plates ${ }^{[36,38]}$. However, with good assay optimization, an acceptable correlation between compound potencies derived from IonWorks assays and manual patch clamp electrophysi- ology can be achieved ${ }^{[36,39]}$. The system can not only be utilized to voltage-gated channels but also for fast-desensitizing ligand-gated channels ${ }^{[38,40]}$. In addition, ion channel-targeted drug-discovery efforts on a number of diverse ion channel subtypes have benefited from the availability of the IonWorks platform with robust assay properties ${ }^{[35,40-46]}$. Thus, the major limitation for Ionworks systems is to establish giga-ohm seals.

To gain a giga-ohm level of seals, several planar array-based automated electrophysiology systems have been developed to incorporate the precision and accuracy of manual electrophysiology recording. The platforms provide a giga-ohm seal with compromised low throughput. However, the platforms have gained prominence and acceptance in the pharmaceutical industry. Some of these platforms are PatchXpress and IonFlux (Molecular Devices, LLC, Sunnyvale, CA, USA), QPatch HT/HTX (Sophion, Copenhagen) ${ }^{[47,48]}$, and Nanion's Patchliner and SynchroPatch (Nanion Technologies GmbH, Munich) ${ }^{[49]}$. The rapid solution exchange time (50-100 ms) and perfusion capability of these systems have made them amenable for ligand-gated ion channel investigations. Furthermore, Nanion's Patchliner offers a primary cell recording capability with both the voltage clamp and current clamp recording modes. Recently, automated patch clamp instruments with a much higher throughput and giga-seal have been launched that bridge the gap between the high-throughput and high quality of ion channel assays, including Sophion's Qube (384well) and Nanion's SyncroPatch 384PE and 768PE.

High-throughput electrophysiology has many theoretical advantages and holds much promise. The continued evolution of existing and new platforms for automated ion channel screening will keep up with the demand both for ion channel safety profiling and for ion channel-targeted drug discovery.

\section{Prevalent HTS methods for the specific ion channel families}

Before choosing the ideal screening method(s), it is important to determine what to look for when comparing technologies and their applications. Eight parameters commonly considered include sensitivity, specificity, throughput, temporal resolution, robustness, flexibility, cost, and physiological relevance. Among all the assay formats of ion channels, undoubtedly, the automated patch clamp assay is the best choice that provides a good quality of data and allows a higher throughput. Currently, an automated electrophysiology assay remains expensive. Thus, not every laboratory can afford it. Therefore, as a compromise, a combination of fluorescence-based screening technologies and an automated patch clamp has become the most commonly used method for ion channel-targeted drug discovery. With cost reduction and technology improvement, automated electrophysiology will become the dominant assay format for most ion channel subtypes. For different ion channel subclasses, the high-throughput screening methods differ due to ion selectivity, channel activation kinetics and a consideration of whether a ligand is needed. For a selected ion channel target, the flowchart of the high-throughput screening is summarized in Figure 1. It is divided into 3 stages: a 
Fluorescence-based assay
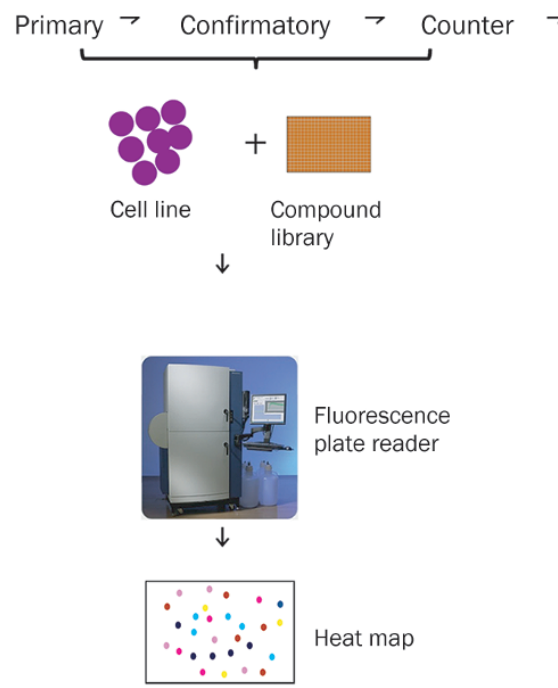

$\downarrow$

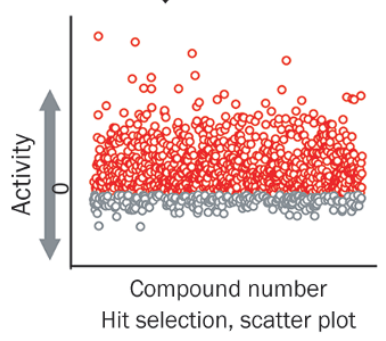

A
Electrophysiological assay

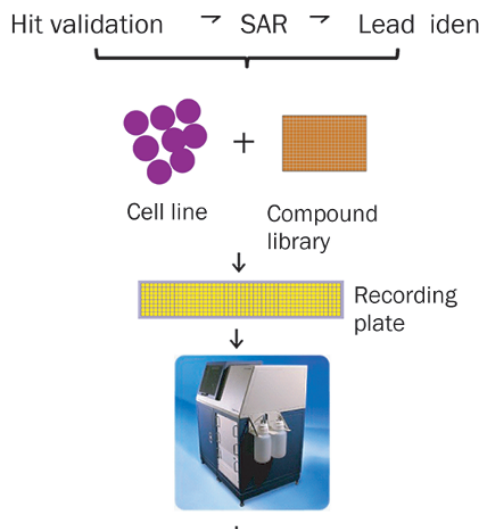

$\downarrow$

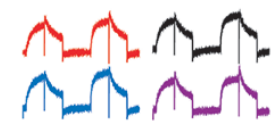

$\downarrow$

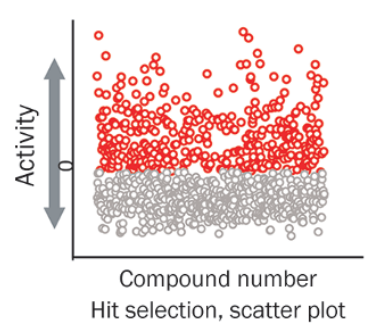

B

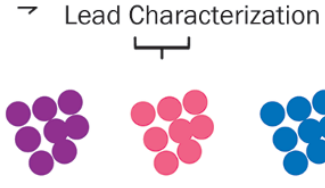

Cell line

Native cells

$\downarrow$

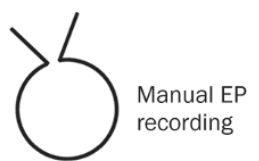

$\downarrow$
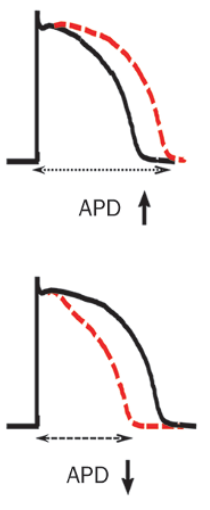

C

Figure 1. Pipeline of high-throughput screening targeting ion channels. (A) For a large compound library, the compounds are first screened using the fluorescence-based flux assay on an ion channel-expressed stable cell line, and the identified hits are confirmed on the same cells and are counterscreened on the parental cells to exclude non-specific hits. (B) Second, active hits are tested using an automated patch clamp for validation and, the active hits are pharmacologically evaluated for structure-activity relationship (SAR) until lead compounds are identified. (C) Finally, a manual patch clamp is used to characterize biophysical properties on stable cell lines and native cells.

fluorescence-based assay for primary screening, an automated patch clamp validation for secondary screening and a manual patch characterization for tertiary screening. Because there are many ion channel families and subclasses available, the most commonly used screening methods will be discussed based on ion selectivity or permeability. The diagrams for the representative screening methods are displayed in Figure 2.

\section{Potassium-selective channels}

Potassium-selective channels are the largest and most diverse group among the ion channel families. The classes of channels include voltage-gated $\left(\mathrm{K}_{\mathrm{v}}\right)$, inward-rectifying $\left(\mathrm{K}_{\mathrm{IR}}\right)$, two-pore $\left(\mathrm{K}_{2 \mathrm{P}}\right)$ and $\mathrm{Ca}^{2+}$-activated $\left(\mathrm{K}_{\mathrm{Ca}}\right)$ potassium channels. Multiple assay formats have been applied to this large family, including the ligand binding assay, ${ }^{86} \mathrm{Rb}^{+}$flux assay, voltage-sensitive dye-assay and $\mathrm{Tl}^{+}$flux assay. Among them, the $\mathrm{Tl}^{+}$flux assay is most often used to identify potassium channel modulators ${ }^{[24]}$. In this assay, $\mathrm{Tl}^{+}$is used as a surrogate ion for $\mathrm{K}^{+}$, and its influx into cells is initially measured using a thalliumsensitive fluorescent dye benzothiazole (BTC $)^{[24,50]}$, which is a
$\mathrm{Ca}^{2+}$ indicator with a low $\mathrm{Ca}^{2+}$-binding affinity $\left(K_{\mathrm{d}}=7 \mu \mathrm{mol} / \mathrm{L}\right)$. Using a commercially available thallium assay kit (FluxOR ${ }^{\mathrm{TM}}$, Life Technologies), the method has been successfully developed for a number of potassium channels. The $\mathrm{Tl}^{+}$flux assay has been extensively used in potassium channel screenings, such as $\mathrm{K}_{\mathrm{v}}^{[24,51-56]}, \mathrm{K}_{\mathrm{IR}}{ }^{[57-59]}, \mathrm{K}_{2 \mathrm{P}}{ }^{[60-62]}$ and $\mathrm{K}_{\mathrm{Ca}}{ }^{[63,64]}$ channels. It should be noted that a variety of off-target pathways (eg, $\mathrm{Na}^{+} / \mathrm{K}^{+}$ATPase) from the native HEK-293 or CHO-K1 (these two parental cell types are most commonly used) cells could interfere with the $\mathrm{Tl}^{+}$influx, which will cause a higher falsepositive or false-negative hit rate. Therefore, a counter screening against parental cells is necessary to eliminate the false hits interacting with parental cells. In addition, the assay should be handled with great care due to toxicity of thallium.

\section{$\mathrm{Ca}^{2+}$-involved ion channels}

An intracellular calcium ion $\left(\mathrm{Ca}^{2+}\right)$ is a universal second messenger that controls both physiological and pathological processes. Among the mentioned ion indicators, calcium indicators are most commonly used ${ }^{[65]}$ because they alter their fluorescence 


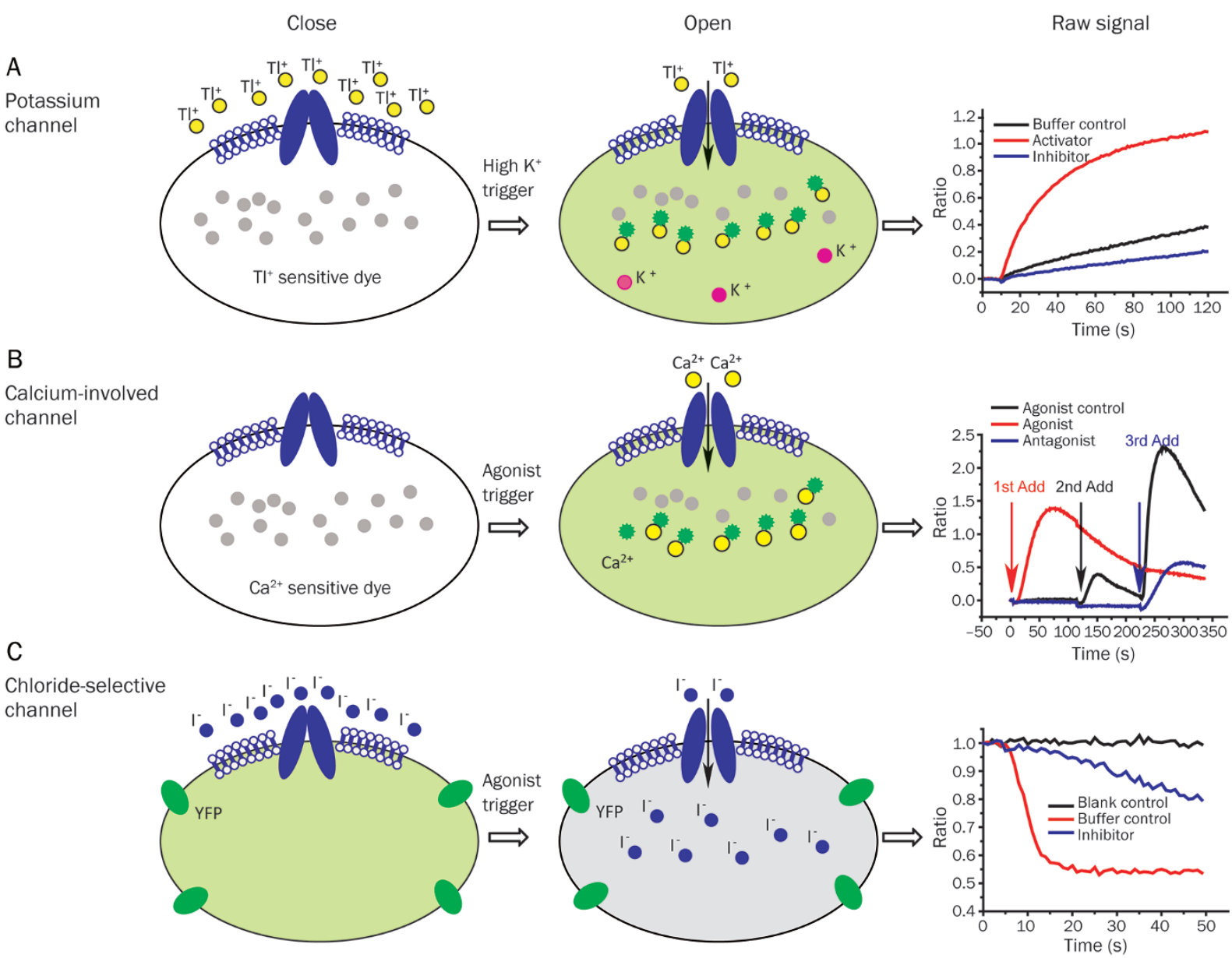

Figure 2. Diagram of high-throughput screening methods. (A) Diagram of the thallium-flux assay for potassium channels, closed state, open state and the raw signal. For a potassium channel, the $\mathrm{Tl}^{+}$-flux assay is the most commonly used assay format. To trigger the channel to open, typically a high- $\mathrm{K}^{+}$ solution is used to depolarize the membrane potential. Then, thallium passes into the cells through open potassium channels. Upon the cytosolic thallium binding to the dye, the fluorescence signal is kinetically increased. The raw traces were acquired from the primary screening of KCNQ2 potassium channels. The black, red and blue raw traces represent the effect of a buffer control, an activator and an inhibitor, respectively. (B) Diagram of calcium-flux assay for calcium-involved channels. Fluo-4 is the most commonly used calcium indicator for calcium-involved channels. To trigger the related channel to open, a high- $\mathrm{Ca}^{2+}$ or a channel agonist is used to open the channel or to increase cytosol $\mathrm{Ca}^{2+}$. The raw data traces were acquired from the primary screening of the TRPC4 channel. To screen for multiple classes of modulators, three additions were applied for the same well. The first addition is for the screened compounds without DAMGO (a highly selective peptide agonist for the $\mu$ opioid receptor) to screen for agonist. The second addition is an $\mathrm{EC}_{20}$ concentration of DAMGO for allosteric modulators. The third addition is an $\mathrm{EC}_{80}$ concentration of DAMGO for antagonists. Black traces represent compounds without any effect to the three additions. Red traces represent compounds with an agonist effect. Blue traces represent compounds with an antagonist effect. (C) Diagram of chloride-selective channels. Mutated YFP (H148Q and I152L) was co-expressed with chloride-selective channels. When the channel opens, iodide enters the cells via chloride channels, binds to YFP and quenches its fluorescence. The raw data were acquired from the primary screening of a calcium-activated chloride channel (TMEM16A). Ionomycin was used as an agonist to activate the channel. Red trace represents the ionomycin-activated channels. Blue trace represents the effect of an inhibitor.

emission upon calcium binding. Currently, over one hundred chemically synthesized and genetically encoded indicators are available ${ }^{[66]}$. Chemical indicators that are most commonly utilized include Fura-2, Indo-1, Fluo-3 and Fluo-4, which are the derivatives of a $\mathrm{Ca}^{2+}$ selective chelator BAPTA ${ }^{[30,67]}$.

Depending on the application, calcium dyes are available in a range of affinities to calcium ions ${ }^{[68]}$, excitation and emission spectra, and chemical forms (membrane permeable or not). They show different temporal resolution (from milliseconds, such as Fluo-3 and Fluo-4, to tens of seconds, such as Fura-
2), and different degrees of accuracy for each range of calcium concentrations. For example, Indo- 1 is preferable to Fluo-3 for measuring large and relatively slow intracellular calcium transients that are associated with cellular contraction. In contrast, Fluo-3 is preferred for measuring small, fast transients that are associated with calcium "sparks" ${ }^{\prime[69]}$. Therefore, Fluo-3 (including its derivative Fluo-4) is more suitable for detecting $\mathrm{Ca}^{2+}$-involved ion channel signals. For the high-throughput screening of $\mathrm{Ca}^{2+}$-involved ion channels, commercial kits, such as the Fluo-4 calcium assay kits (Life Technologies), are avail- 
able. They are extensively applied in $\mathrm{Ca}^{2+}$-involved ion channel assays, such as voltage-gated calcium channels ${ }^{[33,70]}$, TRP channels $^{[71-75]}$, and NMDA receptors ${ }^{[76,77]}$.

Furthermore, calcium channels exist among closed, open and inactivated states. To distinguish the state-dependent inhibitors, usually an inward rectifier potassium channel gene (eg, Kir2.3) is co-expressed with calcium channels. Thus, the membrane potential can be adjusted by altering the external $\mathrm{K}^{+}$concentrations. This approach has successfully been used to identify state-dependent inhibitors and to characterize the molecular selectivity, even offering some advantages over electrophysiology ${ }^{[33,41]}$. The strategy can also be applied to sodium channels. Calcium dye-based ion channel assays suffer from interference from other cellular processes that produce changes in the intracellular calcium concentration. The counter screening for the same assay has to be run against parental cells to remove the nonspecific compounds.

\section{Voltage-gated sodium channels}

Voltage-gated sodium channels are important targets for treating excitable diseases, such as epilepsy and neuropathic pain. It is known that voltage-gated sodium channels exist in closed, open and inactivated states. Additionally, for the sodium channels expressed in HEK-293 cells, the membrane potential is at a more depolarized level. Taking sodium channel subtype Nav1.7 expressed in HEK-293 cells as an example, the averaged membrane potential was measured to be approximately $-24 \mathrm{mV}$, an averaged value from a panel of approximately 50 cells (data not published). Thus, this will drive most channels into inactivated states instead of their real "resting states". Therefore, a known inhibitor of Nav inactivation, veratridine $^{[78]}$, is commonly used to drive the channels open, which produces a more robust signal.

Ideally, ion-selective indicators are perfect to assay the specific ion channels. However, the available $\mathrm{Na}^{+}$sensitive dyes (eg, SBFI ${ }^{[26]}$, CoroNa dyes ${ }^{[79]}$ and Asante Natrium Green$2^{[80]}$ ) are not well-suited for high-throughput screening due to low sensitivity and a poor signal-to-background ratio. The fluorescence-based membrane potential dye assay (eg, DiBAC and FRET dyes) is often used for sodium channel screenings. For the assay itself, the signal change is primarily affected by the membrane potential. Therefore, any event that changes the membrane potential modulates the signals. Thus, the dyebased method may yield a relative high false-positive and/ or false-negative rate when compared with the electrophysiology methods. Recently, a thallium flux $\left(\mathrm{Tl}^{+}\right.$flux)-based method ${ }^{[81]}$, a valuable technique for potassium channels, has been successfully developed as a functional assay for Nav1.7 sodium channels. $\mathrm{Tl}^{+}$flux methods produce dramatically larger signals, which are superior to the state-of-the-art $\mathrm{Na}^{+}-$ sensitive dyes and are amenable for HTS of sodium channels. The application of the ligands (eg, veratridine) may interact with test compounds and thus increase the false-positive or false-negative rates. In addition, a newly developed calcium flux assay (SoCal assay $)^{[82]}$ has been used as a readout of functional change of Nav channels. In this assay, Nav channels were genetically engineered to produce persistent Nav currents with impaired fast inactivation and enhanced calcium permeability. Thus, the calcium reporters, including both the chemical dyes and genetically encoded sensors, would be the alternative indicators of Nav channels. Although the SoCal assay demonstrates a good estimation of activity for the vast majority of tested compounds, it is still recommended that the hits should be further validated using electrophysiological method in wild-type Nav channels. Due to the limitation in controlling the state of sodium channels compared with electrophysiology, care should be taken when interpreting the inhibitor data from the fluorescence-based approach.

\section{Chloride-selective channels}

For chloride channels, the yellow fluorescent protein (YFP) quenching assay has been developed ${ }^{[27]}$. Initially, YFP-H148Q had a higher selectivity for iodide $\left(\mathrm{I}^{-}\right)$than chloride $\left(\mathrm{Cl}^{-}\right)$due to the halide binding properties of $\mathrm{YFP}^{[27]}$. However, H148Q required substantial concentrations of iodide to produce an acceptable signal, which would manifest cell toxicity. A random mutation approach, YFP-I152L, has a significantly increased sensitivity to iodide ${ }^{[27,29]}$. Thus, YFP (H148Q and I152L) combined with iodide was widely used for the chloride channel screening. Upon chloride channel activation, I152L enters the cells, binds to YFP and quenches its fluorescence. Agonist-dependent quench of YFP fluorescence can then be measured with a fluorescence reader and used to determine channel activation, inhibition and modulation. The YFP assay is a noninvasive technique that measures fast responses. The assay has been widely developed for chloride selective channels and receptors, including $\mathrm{CFTR}^{[83,84]}$, calcium-activated $\mathrm{Cl}^{-}$channels (CaCC) (TMEM16A) ${ }^{[85-88]}$, Glycine receptors ${ }^{[89]}$ and GABA receptors ${ }^{[89,90]}$. Additionally, the voltage-sensitive dye method can be used to assay the chloride channels but is rarely used for this class of channels.

Because membrane potential cannot be controlled as electrophysiological assays in the abovementioned fluorescencebased assays, the rationale is to improve the signal-to-noise ratio during the assay development of the HTS. In general, there are two approaches to improve the signal-to-noise ratio: increase the signal or decrease the noise. The signal can be increased by creating conditions under which more channels are opened. This can be achieved by applying high $\mathrm{K}^{+}$to induce membrane depolarization or by applying ligands to drive the channels into more activated states. The noise can be effectively decreased using non-physiological surrogate ions, such as thallium for $\mathrm{K}^{+}$and iodide for $\mathrm{Cl}^{-}$. These surrogate ions are almost non-existent within cells and their noise level is therefore very low. Even a tiny amount intracellular change of these surrogate ions can be easily detected.

\section{Conclusion and perspective}

Overall, progress and improvements in ion channel HTS technologies have sped up ion channel drug discovery. Detection of ion flux signals can be achieved using fluorescence indicator dyes and a fluorescence plate reader, such as the Fluorometric 
Imaging Plate Reader (FLIPR ${ }^{\text {Tetra }}$, Molecular Devices) or FDSS (Hamamatsu Photonics). These assays have relatively low temporal resolution and information content but enable robust and low cost. Electrophysiological methods have the most direct approach to measure ion channel activity and additionally allow flexibility in assay optimization for each channel type. The combination of non-electrophysiological and electrophysiological HTS methods provides an integrated and cost-effective approach for ion channel drug discovery and ensures high quality of data to be generated.

The development of ion channel screening technologies has met most needs for drug discovery. Significant instrumentation development efforts continue to improve the capabilities of automated electrophysiological instruments, which are being used for more ion channel classes and cell types. Emerging trends focus on the exploration of reagents and the development of strategies that may be applied to the screening process of ion channels, including the highly expressed ion channel stable cell lines, sensitive and specific indicators and optimized screening strategies. Expression systems, including offthe-shelf reagents, enable new opportunities for the existing instrumentation. Recently, a number of new products have emerged for the cell line development, including the MaxCyte STX electroporation instrument ${ }^{[91]}$, BacMam system ${ }^{[51]}$ and Jump-In ${ }^{\mathrm{TM}}$ Cell Engineering Platform ${ }^{[92]}$. These products are expected to enhance the stable cell line development process and expedite the screening for drug discovery, bioproduction, and cell-based therapy. Because a constitutive high-level expression of ion channels may cause cell toxicity for some ion channel classes and lead to a drop of the expression level after some passages, the alternative is to use an inducible system that can decrease the toxicity and guarantee long-lasting gene expression. It has been suggested that inducible expression systems should be routinely used because the preparation of a stable cell line is a time-consuming process.

Drug discovery is a slow and complicated process. Identification of the active ingredient is the first and critical step for almost all drug targets. All of the screening-related work is geared towards the first step. Researchers may be interested in searching for multiple classes of chemo-types of ion channel modulators, such as orthosteric modulators, allosteric modulators, or competitive antagonists. Therefore, a flexible design of strategies is encouraged to meet researchers' needs. This flexibility dramatically enhances the possibility of success. Additionally, data analysis and management are also important and critical aspects of the HTS process, especially when analyzing a large amount of data across different assays and targets.

\section{Acknowledgements}

This work was supported by the grants from the National Natural Sciences Foundation of China (Grant № 81470163 and 81503056 to Hai-bo YU) and the National Major Special Project on New Drug Innovation of China (Grant 2014ZX09507003006-008 to Wei-ping WANG).

\section{References}

1 Curran J, Mohler PJ. Alternative paradigms for ion channelopathies: disorders of ion channel membrane trafficking and posttranslational modification. Annu Revi Physiol 2015; 77: 505-24.

2 Kullmann DM, Waxman SG. Neurological channelopathies: new insights into disease mechanisms and ion channel function. J Physiol 2010; 588: 1823-7.

3 Felix R. Channelopathies: ion channel defects linked to heritable clinical disorders. J Med Genet 2000; 37: 729-40.

4 Ptacek LJ. Channelopathies: ion channel disorders of muscle as a paradigm for paroxysmal disorders of the nervous system. Dig Dis Sci 1999; 44: 94S-96S.

5 Bowes J, Brown A, Hamon J, Jarolimek W, Sridhar A, Waldron G, et al. Reducing safety-related drug attrition: the use of in vitro pharmacological profiling. Nat Rev Drug Discov 2012; 11: 909-22.

6 Gill S, Gill R, Lee SS, Hesketh JC, Fedida D, Rezazadeh S, et al. Flux assays in high throughput screening of ion channels in drug discovery. Assay Drug Dev Technol 2003; 1: 709-17.

7 Finlayson K, Pennington AJ, Kelly JS. $\left[{ }^{3} \mathrm{H}\right]$ dofetilide binding in SHSY5Y and HEK293 cells expressing a HERG-like $\mathrm{K}^{+}$channel? Eur J Pharmacol 2001; 412: 203-12.

8 Diaz GJ, Daniell K, Leitza ST, Martin RL, Su Z, McDermott JS, et al. The $\left[{ }^{3} \mathrm{H}\right]$ dofetilide binding assay is a predictive screening tool for hERG blockade and proarrhythmia: Comparison of intact cell and membrane preparations and effects of altering $\left[\mathrm{K}^{+}\right]$. J Pharmacol Toxicol methods 2004; 50: 187-99.

9 Chiu PJ, Marcoe KF, Bounds SE, Lin CH, Feng JJ, Lin A, et al. Validation of a $\left[{ }^{3} \mathrm{H}\right]$ astemizole binding assay in HEK293 cells expressing HERG $\mathrm{K}^{+}$channels. J Pharmacol Sci 2004; 95: 311-9.

10 Huang XP, Mangano T, Hufeisen S, Setola V, Roth BL. Identification of human Ether-à-go-go related gene modulators by three screening platforms in an academic drug-discovery setting. Assay Drug Dev Technol 2010; 8: 727-42.

11 Hamilton TC, Weir SW, Weston AH. Comparison of the effects of BRL 34915 and verapamil on electrical and mechanical activity in rat portal vein. Br J Pharmacol 1986; 88: 103-11.

12 Weir SW, Weston AH. The effects of BRL 34915 and nicorandil on electrical and mechanical activity and on $86 \mathrm{Rb}$ efflux in rat blood vessels. Br J Pharmacol 1986; 88: 121-8.

13 Terstappen GC. Functional analysis of native and recombinant ion channels using a high-capacity nonradioactive rubidium efflux assay. Anal Biochem 1999; 272: 149-55.

14 Liu K, Samuel M, Harrison RK, Paslay JW. Rb ${ }^{+}$efflux assay for assessment of non-selective cation channel activities. Assay Drug Dev Technol 2010; 8: 380-8.

15 Chaudhary KW, O'Neal JM, Mo ZL, Fermini B, Gallavan RH, Bahinski A. Evaluation of the rubidium efflux assay for preclinical identification of HERG blockade. Assay Drug Dev Technol 2006; 4: 73-82.

16 Scott CW, Wilkins DE, Trivedi S, Crankshaw DJ. A mediumthroughput functional assay of KCNQ2 potassium channels using rubidium efflux and atomic absorption spectrometry. Anal Biochem 2003; 319: 251-7.

17 Terstappen GC. Nonradioactive rubidium ion efflux assay and its applications in drug discovery and development. Assay Drug Dev Technol 2004; 2: 553-9.

18 Epps DE, Knechtel TJ, Bacznskyj O, Decker D, Guido DM, Buxser SE, et al. Tirilazad mesylate protects stored erythrocytes against osmotic fragility. Chem Phys Lipids 1994; 74: 163-74.

19 Gonzalez JE, Tsien RY. Voltage sensing by fluorescence resonance energy transfer in single cells. Biophys J 1995; 69: 1272-80. 
20 Whiteaker KL, Gopalakrishnan SM, Groebe D, Shieh CC, Warrior U, Burns DJ, et al. Validation of FLIPR membrane potential dye for high throughput screening of potassium channel modulators. J Biomol Screen 2001; 6: 305-12.

21 Kao JP, Harootunian AT, Tsien RY. Photochemically generated cytosolic calcium pulses and their detection by fluo-3. J Biol Chem 1989; 264 : 8179-84.

22 Minta A, Kao JP, Tsien RY. Fluorescent indicators for cytosolic calcium based on rhodamine and fluorescein chromophores. J Biol Chem 1989; 264: 8171-8.

23 Beacham DW, Blackmer T, M OG, Hanson GT. Cell-based potassium ion channel screening using the FluxOR assay. J Biomol Screen 2010; 15: 441-6.

24 Weaver CD, Harden D, Dworetzky SI, Robertson B, Knox RJ. A thallium-sensitive, fluorescence-based assay for detecting and characterizing potassium channel modulators in mammalian cells. J Biomol Screen 2004; 9: 671-7.

25 Meuwis K, Boens N, De Schryver FC, Gallay J, Vincent M. Photophysics of the fluorescent $\mathrm{K}^{+}$indicator PBFI. Biophys J 1995; 68: 2469-73.

26 Minta A, Tsien RY. Fluorescent indicators for cytosolic sodium. Biol Chem 1989; 264: 19449-57.

27 Galietta $\sqcup$, Haggie PM, Verkman AS. Green fluorescent protein-based halide indicators with improved chloride and iodide affinities. FEBS lett 2001; 499: 220-4.

28 Wachter RM, Remington SJ. Sensitivity of the yellow variant of green fluorescent protein to halides and nitrate. Curr Biol 1999; 9: R628-9.

29 Jayaraman S, Haggie P, Wachter RM, Remington SJ, Verkman AS. Mechanism and cellular applications of a green fluorescent proteinbased halide sensor. J Biol Chem 2000; 275: 6047-50.

30 Grynkiewicz G, Poenie M, Tsien RY. A new generation of $\mathrm{Ca}^{2+}$ indicators with greatly improved fluorescence properties. J Biol Chem 1985; 260: 3440-50.

31 Song Y, Buelow B, Perraud AL, Scharenberg AM. Development and validation of a cell-based high-throughput screening assay for TRPM2 channel modulators. J Biomol Screen 2008; 13: 54-61.

32 Miller M, Shi J, Zhu Y, Kustov M, Tian JB, Stevens A, et al. Identification of ML204, a novel potent antagonist that selectively modulates native TRPC4/C5 ion channels. J Biol Chem 2011; 286: 33436-46.

33 Dai G, Haedo RJ, Warren VA, Ratliff KS, Bugianesi RM, Rush A, et al. A high-throughput assay for evaluating state dependence and subtype selectivity of Cav2 calcium channel inhibitors. Assay Drug Dev Technol 2008; 6: 195-212.

34 Finkel A, Wittel A, Yang N, Handran S, Hughes J, Costantin J. Population patch clamp improves data consistency and success rates in the measurement of ionic currents. J Biomol Screen 2006; 11 : 488-96.

35 Trivedi S, Dekermendjian K, Julien R, Huang J, Lund PE, Krupp J, et al. Cellular HTS assays for pharmacological characterization of $\mathrm{Na}(\mathrm{V}) 1.7$ modulators. Assay Drug Dev Technol 2008; 6: 167-79.

36 Bridgland-Taylor MH, Hargreaves AC, Easter A, Orme A, Henthorn DC, Ding $\mathrm{M}$, et al. Optimisation and validation of a medium-throughput electrophysiology-based hERG assay using lonWorks HT. J Pharmacol Toxicol Methods 2006; 54: 189-99.

37 Wible BA, Kuryshev YA, Smith SS, Liu Z, Brown AM. An ion channel library for drug discovery and safety screening on automated platforms. Assay Drug Dev Technol 2008; 6: 765-80.

38 Gillie DJ, Novick SJ, Donovan BT, Payne LA, Townsend C. Development of a high-throughput electrophysiological assay for the human ether-ago-go related potassium channel hERG. J Pharmacol Toxicol Methods
2013; 67: 33-44.

39 Terstappen GC, Roncarati R, Dunlop J, Peri R. Screening technologies for ion channel drug discovery. Future Med Chem 2010; 2: 715-30.

40 Graef JD, Benson LC, Sidach SS, Wei H, Lippiello PM, Bencherif M, et al. Validation of a high-throughput, automated electrophysiology platform for the screening of nicotinic agonists and antagonists. J Biomol Screen 2013; 18: 116-27.

41 Xie X, Van Deusen AL, Vitko I, Babu DA, Davies LA, Huynh N, et al. Validation of high throughput screening assays against three subtypes of $\mathrm{Ca}(\mathrm{v}) 3 \mathrm{~T}$-type channels using molecular and pharmacologic approaches. Assay Drug Dev Technol 2007; 5: 191-203.

42 Lee YT, Vasilyev DV, Shan QJ, Dunlop J, Mayer S, Bowlby MR. Novel pharmacological activity of loperamide and CP-339,818 on human HCN channels characterized with an automated electrophysiology assay. Eur J Pharmacol 2008; 581: 97-104.

43 Castle N, Printzenhoff D, Zellmer S, Antonio B, Wickenden A, Silvia C. Sodium channel inhibitor drug discovery using automated high throughput electrophysiology platforms. Comb Chem High Throughput Screen 2009; 12: 107-22.

44 Ido K, Ohwada T, Yasutomi E, Yoshinaga T, Arai T, Kato M, et al. Screening quality for $\mathrm{Ca}^{2+}$-activated potassium channel in lonWorks Quattro is greatly improved by using BAPTA-AM and ionomycin. J Pharmacol Toxicol Methods 2013 67: 16-24.

45 Du F, Yu H, Zou B, Babcock J, Long S, Li M. hERGCentral: a large database to store, retrieve, and analyze compound-human Ether-ago-go related gene channel interactions to facilitate cardiotoxicity assessment in drug development. Assay Drug Dev Technol 2011; 9: $580-8$

46 Zhang H, Zou B, Yu H, Moretti A, Wang X, Yan W, et al. Modulation of hERG potassium channel gating normalizes action potential duration prolonged by dysfunctional KCNQ1 potassium channel. Proc Natl Acad Sci U S A 2012; 109: 11866-71.

47 Beck EJ, Hutchinson TL, Qin N, Flores CM, Liu Y. Development and validation of a secondary screening assay for TRPM8 antagonists using QPatch HT. Assay Drug Dev Technol 2010; 8: 63-72.

48 Liu Y, Beck EJ, Flores CM. Validation of a patch clamp screening protocol that simultaneously measures compound activity in multiple states of the voltage-gated sodium channel Nav1.2. Assay Drug Dev Technol 2011; 9: 628-34.

49 Milligan CJ, Li J, Sukumar P, Majeed Y, Dallas ML, English A, et al. Robotic multiwell planar patch-clamp for native and primary mammalian cells. Nat Protoc 2009; 4: 244-55.

50 Hyrc KL, Bownik JM, Goldberg MP. Ionic selectivity of low-affinity ratiometric calcium indicators: mag-Fura-2, Fura-2FF and BTC. Cell calcium 2000; 27: 75-86.

51 Titus SA, Beacham D, Shahane SA, Southall N, Xia M, Huang R, et al. A new homogeneous high-throughput screening assay for profiling compound activity on the human ether-a-go-go-related gene channel. Anal Biochem 2009; 394: 30-8.

52 Cheung YY, Yu H, Xu K, Zou B, Wu M, McManus OB, et al. Discovery of a series of 2-phenyl-N-(2-(pyrrolidin-1-yl)phenyl)acetamides as novel molecular switches that modulate modes of K(v)7.2 (KCNQ2) channel pharmacology: identification of (S)-2-phenyl-N-(2-(pyrrolidin-1-yl)phenyl)butanamide (ML252) as a potent, brain penetrant $\mathrm{K}(\mathrm{v}) 7.2$ channel inhibitor. J Med Chem 2012; 55: 6975-9.

53 Yu H, Wu M, Townsend SD, Zou B, Long S, Daniels JS, et al. Discovery, Synthesis, and Structure Activity Relationship of a Series of N-Arylbicyclo[2.2.1]heptane-2-carboxamides: Characterization of ML213 as a Novel KCNQ2 and KCNQ4 Potassium Channel Opener. ACS Chem Neurosci 2011; 2: 572-77.

54 Zou B, Yu H, Babcock JJ, Chanda P, Bader JS, McManus OB, et al. Pro- 
filing diverse compounds by flux- and electrophysiology-based primary screens for inhibition of human Ether-a-go-go related gene potassium channels. Assay Drug Dev Technol 2010; 8: 743-54.

55 Mattmann ME, Yu H, Lin Z, Xu K, Huang X, Long S, et al. Identification of (R)-N-(4-(4-methoxyphenyl)thiazol-2-yl)-1-tosylpiperidine2-carboxamide, ML277, as a novel, potent and selective $\mathrm{K}(\mathrm{v}) 7.1$ (KCNQ1) potassium channel activator. Bioorg Med Chem Lett 2012; 22: 5936-41.

56 Bridal TR, Margulis M, Wang X, Donio M, Sorota S. Comparison of human Ether-a-go-go related gene screening assays based on lonWorks Quattro and thallium flux. Assay Drug Dev Technol 2010; 8: 755-65.

57 Wang HR, Wu M, Yu H, Long S, Stevens A, Engers DW, et al. Selective inhibition of the K(ir)2 family of inward rectifier potassium channels by a small molecule probe: the discovery, SAR, and pharmacological characterization of ML133. ACS Chem Biol 2011; 6: 845-56.

58 Raphemot R, Kadakia RJ, Olsen ML, Banerjee S, Days E, Smith SS, et al. Development and validation of fluorescence-based and automated patch clamp-based functional assays for the inward rectifier potassium channel Kir4.1. Assay Drug Dev Technol 2013; 11: 532-43.

59 Raphemot R, Weaver CD, Denton JS. High-throughput screening for small-molecule modulators of inward rectifier potassium channels. J Vis Exp 2013; 71: e4209.

60 Flaherty DP, Simpson DS, Miller M, Maki BE, Zou B, Shi J, et al. Potent and selective inhibitors of the TASK-1 potassium channel through chemical optimization of a bis-amide scaffold. Bioorg Med Chem Lett 2014; 24: 3968-73.

61 Bruner JK, Zou B, Zhang H, Zhang Y, Schmidt K, Li M. Identification of novel small molecule modulators of K2P18.1 two-pore potassium channel. Eur J Pharmacol 2014; 740: 603-10.

62 Zou B, Flaherty DP, Simpson DS, Maki BE, Miller MR, Shi J, et al. ML365: Development of bis-amides as selective inhibitors of the KCNK3/TASK1 two pore potassium channel. In: Probe Reports from the NIH Molecular Libraries Program. Bethesda (MD): National Center for Biotechnology Information (US); 2010.

63 Jorgensen S, Dyhring T, Brown DT, Strobaek D, Christophersen P, Demnitz J. A high-throughput screening campaign for detection of $\mathrm{Ca}^{2+}$ activated $\mathrm{K}^{+}$channel activators and inhibitors using a fluorometric imaging plate reader-based $\mathrm{TI}^{+}$-influx assay. Assay Drug Dev Technol 2013; 11: 163-72.

64 Jorgensen S, Johansen TH, Dyhring T. Fluorescence-based $\mathrm{TI}^{+}$-influx assays as a novel approach for characterization of small-conductance $\mathrm{Ca}^{2+}$-activated $\mathrm{K}^{+}$channel modulators. Methods Mol Biol 2008; 491: 257-66.

65 Hanson GT, Hanson BJ. Fluorescent probes for cellular assays. Comb Chem High Throughput Screen 2008; 11: 505-13.

66 Horikawa K. Recent progress in the development of genetically encoded $\mathrm{Ca}^{2+}$ indicators. J Med Invest 2015; 62: 24-8.

67 Tsien RY. New calcium indicators and buffers with high selectivity against magnesium and protons: design, synthesis, and properties of prototype structures. Biochemistry 1980; 19: 2396-404.

68 Paredes RM, Etzler JC, Watts LT, Zheng W, Lechleiter JD. Chemical calcium indicators. Methods 2008; 46: 143-51.

69 Bailey S, Macardle PJ. A flow cytometric comparison of Indo-1 to fluo3 and Fura Red excited with low power lasers for detecting $\mathrm{Ca}^{2+}$ flux. J Immunol Methods 2006; 311: 220-5.

70 Belardetti F, Tringham E, Eduljee C, Jiang X, Dong H, Hendricson A, et al. A fluorescence-based high-throughput screening assay for the identification of T-type calcium channel blockers. Assay Drug Dev Technol 2009; 7: 266-80.

71 Bandell M, Dubin AE, Petrus MJ, Orth A, Mathur J, Hwang SW, et al. High-throughput random mutagenesis screen reveals TRPM8 residues specifically required for activation by menthol. Nat Neurosci 2006; 9: 493-500.

72 Castillo B, Porzgen P, Penner R, Horgen FD, Fleig A. Development and optimization of a high-throughput bioassay for TRPM7 ion channel inhibitors. J Biomol Screen 2010; 15: 498-507.

73 Miller M, Wu M, Xu J, Weaver D, Li M, Zhu MX. High-Throughput Screening of TRPC Channel Ligands Using Cell-Based Assays. In: Zhu MX, editor. TRP Channels. Boca Raton (FL): CRC Press/Taylor \& Francis; 2011. Chapter1.

74 Zicha S, Radresa O, Laplante P, Morton M, Jones K, Main M, et al. Novel methodology to identify TRPV1 antagonists independent of capsaicin activation. J Biomol Screen 2013; 18: 544-55.

75 Luo J, Zhu Y, Zhu MX, Hu H. Cell-based calcium assay for medium to high throughput screening of TRP channel functions using FlexStation 3. J Vis Exp 2011; 54: e3149.

76 Bettini E, Sava A, Griffante C, Carignani C, Buson A, Capelli AM, et al. Identification and characterization of novel NMDA receptor antagonists selective for NR2A- over NR2B-containing receptors. J Pharmacol Exp Ther 2010; 335: 636-44.

77 Davies DJ, Crowe M, Lucas N, Quinn J, Miller DD, Pritchard S, et al. A novel series of benzimidazole NR2B-selective NMDA receptor antagonists. Bioorg Med Chem Lett 2012; 22: 2620-3.

78 Benjamin ER, Pruthi F, Olanrewaju S, Ilyin VI, Crumley G, Kutlina E, et al. State-dependent compound inhibition of Nav1.2 sodium channels using the FLIPR Vm dye: on-target and off-target effects of diverse pharmacological agents. J Biomol Screen 2006; 11: 29-39.

79 Diaz P, D'Suze G, Salazar V, Sevcik C, Shannon JD, Sherman NE, et al. Antibacterial activity of six novel peptides from Tityus discrepans scorpion venom. A fluorescent probe study of microbial membrane $\mathrm{Na}^{+}$permeability changes. Toxicon 2009; 54: 802-17.

80 Roder P, Hille C. ANG-2 for quantitative $\mathrm{Na}^{+}$determination in living cells by time-resolved fluorescence microscopy. Photochem Photobiol Sci 2014; 13: 1699-710.

81 Du Y, Days E, Romaine I, Abney KK, Kaufmann K, Sulikowski G, et al. Development and Validation of a Thallium Flux-Based Functional Assay for the Sodium Channel NaV1.7 and Its Utility for Lead Discovery and Compound Profiling. ACS Chem Neurosci 2015; 6: 871-8.

82 Zhang H, Zou B, Du F, Xu K, Li M. Reporting sodium channel activity using calcium flux: pharmacological promiscuity of cardiac Nav1.5. Mol Pharmacol 2015; 87: 207-17.

83 Sui J, Cotard S, Andersen J, Zhu P, Staunton J, Lee M, et al. Optimization of a Yellow fluorescent protein-based iodide influx high-throughput screening assay for cystic fibrosis transmembrane conductance regulator (CFTR) modulators. Assay Drug Dev Technol 2010; 8: 656-68.

84 Galietta L, Springsteel MF, Eda M, Niedzinski EJ, By K, Haddadin MJ, et al. Novel CFTR chloride channel activators identified by screening of combinatorial libraries based on flavone and benzoquinolizinium lead compounds. J Biol Chem 2001; 276: 19723-8.

85 Namkung W, Phuan PW, Verkman AS. TMEM16A inhibitors reveal TMEM16A as a minor component of calcium-activated chloride channel conductance in airway and intestinal epithelial cells. J Biol Chem 2011; 286: 2365-74.

86 Namkung W, Thiagarajah JR, Phuan PW, Verkman AS. Inhibition of $\mathrm{Ca}^{2+}$-activated $\mathrm{Cl}$ - channels by gallotannins as a possible molecular basis for health benefits of red wine and green tea. Faseb J 2010; 24 : 4178-86.

87 Namkung W, Yao Z, Finkbeiner WE, Verkman AS. Small-molecule activators of TMEM16A, a calcium-activated chloride channel, stimulate epithelial chloride secretion and intestinal contraction. Faseb J 2011; 25: 4048-62.

88 Peters CJ, Yu H, Tien J, Jan YN, Li M, Jan LY. Four basic residues criti- 
cal for the ion selectivity and pore blocker sensitivity of TMEM16A calcium-activated chloride channels. Proc Natl Acad Sci U S A 2015; 112: 3547-52.

89 Kruger W, Gilbert D, Hawthorne R, Hryciw DH, Frings S, Poronnik P, et al. A yellow fluorescent protein-based assay for high-throughput screening of glycine and GABAA receptor chloride channels. Neurosci Lett 2005; 380: 340-5.

90 Johansson T, Norris T, Peilot-Sjogren H. Yellow Fluorescent ProteinBased Assay to Measure GABAA Channel Activation and Allosteric
Modulation in CHO-K1 Cells. PloS One 2013; 8: e59429.

91 Johansson T, Norris T, Peilot-Sjogren H. Yellow fluorescent proteinbased assay to measure GABA(A) channel activation and allosteric modulation in CHO-K1 cells. PloS One 2013; 8: e59429.

92 Lieu PT, Machleidt T, Thyagarajan B, Fontes A, Frey E, Fuerstenau-Sharp $M$, et al. Generation of site-specific retargeting platform cell lines for drug discovery using phiC31 and R4 integrases. J Biomol Screen 2009; 14: 1207-15. 